\title{
Formation of the Planetary Candidates Observed by Kepler Mission
}

\author{
Su Wang and Jianghui Ji \\ Key Laboratory of Planetary Sciences, Purple Mountain Observatory, \\ Chinese Academy of Sciences, Nanjing 210008, China \\ email: wangsu@pmo.ac.cn, jijh@pmo.ac.cn
}

\begin{abstract}
The Kepler Mission which launched in 2009 March focuses on detecting potentially habitable terrestrial-sized planets. To date the Kepler mission has released more than 4000 planetary candidates. There are plenty of planet pairs trapped near the first order mean motion resonance (MMR). From the statistical results of numerical simulations based on the formation scenario we proposed for the planetary configurations near 3:2 and 2:1 MMRs, we find that the proportions of period ratios close to 1.5 and 2.0 can arrive at $14.5 \%$ and $26.0 \%$, respectively. This scenario may explain the formation of Kepler candidates pairs in near 3:2 and 2:1 MMRs.
\end{abstract}

Keywords. (stars:) planetary systems: formation, methods: numerical

\section{Introduction}

Up to 2015 September, there are $\sim 4696$ planetary candidates with $\sim 1889$ confirmed planets released from the Kepler mission data. Among them, 473 multiple planetary systems exist. The analysis results on the planets from the first 16 months released show that plenty planet pairs are near MMRs in 222 three-planet systems (Fabrycky et al. 2014) especially the first orders, $\sim 7.0 \%$ near 3:2 MMR (the period ratio in the range of $[1.45,1.54]$ ) and $\sim 18.0 \%$ near 2:1 MMR (the period ratio in the range of $[1.83,2.18]$ ). Near 4:2:1 and 3:2:1 MMRs chains may appear in multiple planetary systems.

Wang et al. (2012) has explained the formation of planetary system KOI-152 which contains three planets in near 4:2:1 MMRs. The formation scenario of such system is supposed as: firstly, planets form far away from the central star; then they undergo orbital migration due to the effect of the gas disk until they go to the inner region of the system, in the process they are trapped into MMRs; at last, with the tidal effect raised by the central star they are out of MMRs to be a configuration similar to KOI-152 with three planets in near 4:2:1 MMRs (Terquem \& Papaloizou 2007, Lithwick \& Wu 2012, Wang et al. 2012, Batygin \& Morbidelli 2013, Delisle \& Laskar 2014, Wang \& Ji 2014). The formation scenario may explain the formation of planet pairs near 3:2 and 2:1 MMRs. The properties of star such as the accretion rate $(\dot{M})$ and the magnetic field $\left(B_{*}\right)$, the disk model especially the gas density profile, the speed of orbital migration (with a reduce factor $f_{1}$ of type I migration), the masses $(m)$ and numbers of planets in the system are the main factors that influence the formation of final planetary system configuration.

\section{Numerical simulations and statistical results}

In the numerical simulation model, we consider a solar-like star with three planets (P1 represents the innermost one, P2 is the middle planet, and P3 displays the outmost one) 
around in the system. All three planets embed in the gas disk initially. There are four groups of simulations with different masses of planets. According to the isolation mass (Ida \& Lin 2004), the mass of planet is proportional to $a^{3 / 4}$, where $a$ is the semi-major axis of planet. Thus, in the first group, we choose the masses of three planets increased in turn, they are $m_{p 1}=5 M_{\oplus}, m_{p 2}=10 M_{\oplus}$, and $m_{p 3}=15 M_{\oplus}$, respectively. In the second group, we set planets with two kinds of masses: three planets with equal masses 5 $M_{\oplus}$, and $m_{p 1}=m_{p 2}=5 M_{\oplus}$ with an outer larger planet $m_{p 3}=10 M_{\oplus}$. We also consider the masses of planets in other two groups based on the statistical results on planetary candidates from the Kepler data. The masses ratios are 1:1:1.5, 2:1:7.5, 12:1:0.2, and $2: 0.2: 4$, respectively. In the simulations, the star accretion rate is in the range of $[0.1,2.5]$ $\times 10^{-8} M_{\odot} \mathrm{yr}^{-1}$, the magnetic field of star is from 0.5 to $2.5 \mathrm{KG}$, the reduction factor of type I migration $f_{1}$ is chosen to be $0.01,0.03,0.1,0.3$, and 1 . Three planets are at the orbital periods of 100,250 , and 600 days for $f_{1}=0.01$ and 0.03 , while they are at 140 , 500 , and 1450 days for $f_{1} \geqslant 0.1$ at the beginning of the simulations. Initially all three planets are in coplanar and near-circular orbits, the mean anomaly, the argument of the pericenter, and the longitude of ascending node are generated randomly in the range of $\left[0^{\circ}, 360^{\circ}\right]$. The gas disk profile is $\Sigma_{g} \propto r^{-1}$ (Pringle 1981) and will deplete in a timescale of $10^{6} \mathrm{yr}$. In the disk, there are two locations with gas density changed sharply and the locations are related to the magnetic field and the star accretion rate. Migrating planets may be stopped at these two locations (Masset et al. 2006, Kretke et al. 2009, Wang et al. 2012). In our simulations, the masses of all planets are less than $100 M_{\oplus}$, besides the gravitational effects among them, they suffer type I migration and the gas damping force before the gas dissipated.

We carried out 1020 runs of simulations with different parameters $\left(\dot{M}, B_{*}, f_{1}, m\right)$ and potentially planets survived in the systems. The main results are shown in Figure 1. Panel (a) shows the overall results from the simulations compared with the observation. In both data, two peaks are obtained and when $\dot{M}=1 \times 10^{-9} \mathrm{M}_{\odot} \mathrm{yr}^{-1}$ they have similar distribution roughly. Panel (b1) and (b2) show the fraction of planet pairs near 2:1 or 3:2 MMRs changing with the star accretion rate and migration speed, respectively. The early stage of star with high accretion rate, $\dot{M}=2 \times 10^{-8} \mathrm{M}_{\odot} \mathrm{yr}^{-1}$, is good to the formation of near 2:1 MMRs, while the late stage may be responsible for the formation of near $3: 2$ MMRs, $\dot{M}=0.1 \times 10^{-8} \mathrm{M}_{\odot} \mathrm{yr}^{-1} . f_{1} \geqq 0.1$ and $f_{1} \geqq 0.3$ favor the formation of near 2:1 and 3:2 MMRs, respectively. In our simulations, we also test the influence of additional planets which potentially survive in the system. Panel (c1) and (c2) show the main results. In panel (c1), if there are three planets P1, P2, and P3 in the system, they will be in near 4:2:1MMRs at the end of the simulation, here shows the result that there are additional planet E1 locating inside the planet $\mathrm{P} 1$ and E2 residing outside the planet P3 with other conditions the same with the previous simulation, 2:1 MMRs are not affected, the Laplacian resonance is not disrupted easily. But in panel (c2) for the system which are involved in two pairs of 3:2 MMRs for only three planets exist, the results are very different, 3:2 MMRs will disintegrate at $\sim 0.2 \mathrm{Myr}$.

\section{Discussion}

Using the formation scenario we supposed, the peak near 2:1 and 3:2 MMRs can be obtained. The near 4:2:1 MMRs are relatively stable, such configuration may be common in the exoplanetary systems. The near 3:2:1 MMRs may be appeared in the system with more than three planets. In the future work, we will consider the effect of mass growing process on the formation scenario and the configuration formation with giant planets in the system. 

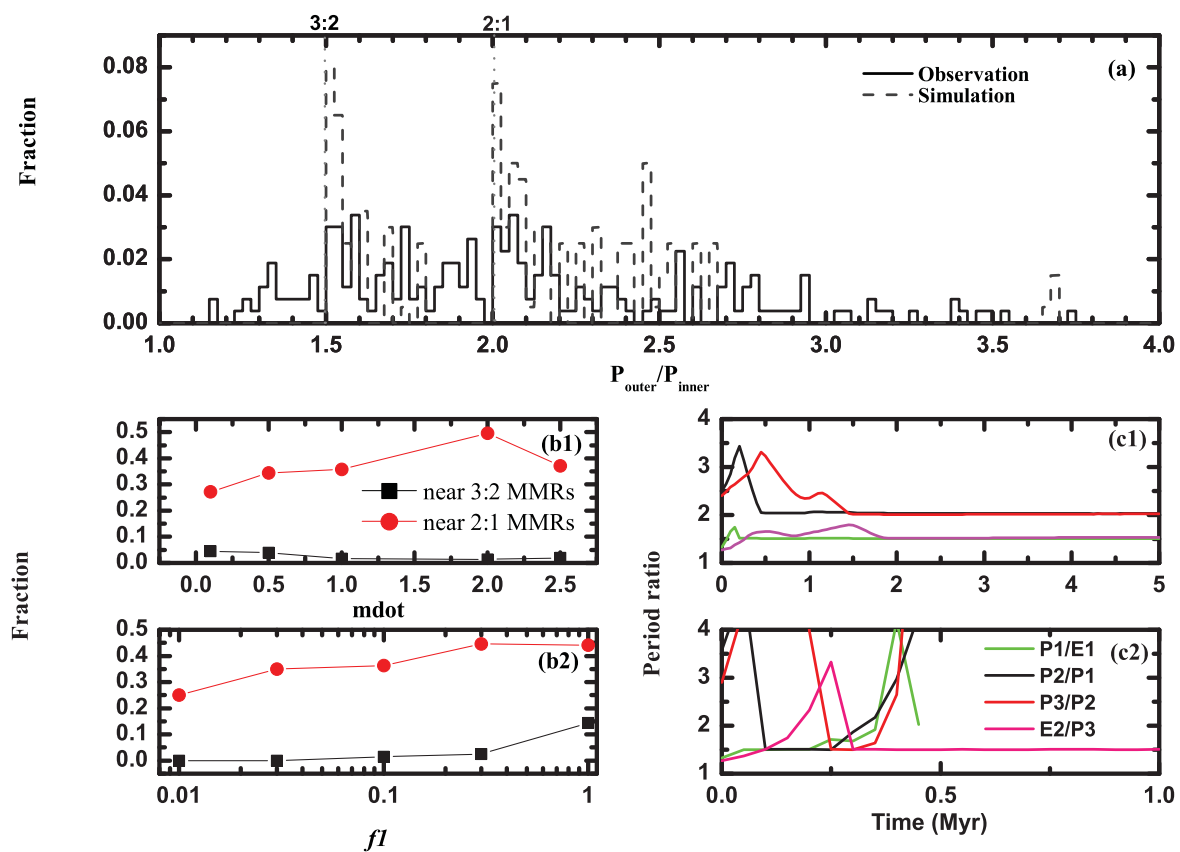

Figure 1. (a) The statistical results of the simulations compared with the observation data. The solid line displays the observation results and the dashed line represents the simulation results. (b1) and (b2) show the fraction that two planets pair in 2:1 MMRs or 3:2 MMRs differs with the star accretion rate and the orbital migration speed, respectively. (c1) The effect of additional planets on the system with planet pairs which would in 2:1 MMRs if there are only three planets in the system. (c2) The effect of additional planets on the system with planet pairs which would in 3:2 MMRs if there are only three planets in the system.

\section{Acknowledgements}

This work is financially supported by National Natural Science Foundation of China (Grants No. 11203087, 11573073, 11273068), the Natural Science Foundation of Jiangsu Province (Grant No. BK20151607), the Strategic Priority Research Program on Space Science (Grant No. XDA04060901), and the Foundation of Minor Planets of Purple Mountain Observatory.

\section{References}

Batygin, K. \& Morbidelli, A. 2013, AJ, 145, 1

Delisle, J. B. \& Laskar, J. 2014, A\&SA, 570, L7

Fabrycky, D. C., Lissauer, J. J., Ragozzine, D., et al. 2014, ApJ, 790, 146

Ida, S. \& Lin, D. N. C. $2004, A p J, 604,388$

Kretke, K. A. et al. 2009, ApJ, 690, 407

Lithwick, Y. \& Wu, Y. 2012, ApJL, 756, 11

Masset F. S. et al. 2006, ApJ, 642, 478

Pringle, J. E. 1981, ARA \& A, 19, 137

Terquem, C. \& Paploizou, J. C. B. 2007, ApJ, 654, 1110

Wang, S., Ji, J. H., \& Zhou, J. L. 2012, ApJ, 753, 170

Wang, S. \& Ji J. H. 2014, ApJ, 795, 85 